\title{
Psychiatric morbidity in a case of encephalomalacia
}

\author{
Jnanamay Das ${ }^{1, *}$, Shailly Yadav $^{2}$ \\ ${ }^{1}$ Specialist and Head, ${ }^{2}$ Senior Resident, Dept. of Psychiatry, ESI Hospital, Rohini, New Delhi, India \\ *Corresponding Author: \\ Email: drjdas@hotmail.com
}

\begin{abstract}
We report a case of an adult female who had changes suggestive of encephalomalacia in the magnetic resonance imaging (MRI) of the brain but presented with psychiatric symptomatology instead of neurological manifestations. Encephalomalacia is softening of brain tissue which may lead to brain changes and present with varied clinical manifestations. Most of the cases reported previously were on infants \& children and almost all of them were related to neurological disorders. But cases with psychiatric symptomatology were rarely reported, that too in adults. The authors discussed the psychiatric symptom profile, their management and emphasized the importance of imaging of the brain and its association with psychiatric manifestations.
\end{abstract}

Keywords: Encephalomalacia, Psychiatric morbidity, Brain imaging.

\section{Introduction}

Encephalomalacia is the softening or loss of brain tissue after cerebral infarction, cerebral ischemia, infection, craniocerebral trauma, or other injury. ${ }^{1}$ In the imaging classification of traumatic brain injury, encephalomalacia is a type of chronic condition secondary to injury of the brain. ${ }^{2}$ Cerebral softening leads to brain changes which can have varied clinical manifestations. Though very few published data on encephalomalacia are available in case of human beings most of the articles are in infants and children but rarely in adults. ${ }^{3}$ Almost all of them are related to neurological and/or seizure disorders. ${ }^{3-5}$ In adults with encephalomalacia, rare case reports are available who presented with psychiatric morbidities in the form of progressive mental decline, borderline dementia, features of depression, delusion and oedipism. ${ }^{6,7}$ Here we present a case of encephalomalacia in an adult female who presented with psychiatric symptomatology.

\section{Case Report}

Mrs. ND, a 36-year-old house wife and a mother of three children from a lower socio-economic background, was brought to psychiatry OPD by her husband with the complaints of feeling low, headache, anxiety, fatiguability, decreased concentration, decreased sleep and decreased appetite for one year. The symptoms had a gradual onset. She remained sad most of the time. She had difficulty in falling asleep, she would sleep only for 3-4 hours at night and would often wake up midnight. She would feel tired throughout the day. Her interest in household work decreased, she was getting no interest in watching her favourite television shows which she used to enjoy before. She felt as if she had decreased energy from inside and perceived doing her routine chores as a burden on her. Her interaction with her family members also decreased and she used to get irritable easily on trivial issues. She described her headache as a dull and aching type of pain involving the whole head, occurring almost daily with mild fluctuations. There was relief in headache by taking rest. She won't feel like having food. She would always worry about her family members that something terrible might happen to them.

There was no precipitating factor. There was no history suggestive of any suicidal ideas, obsession, compulsion, phobia, photophobia, phonophobia, unconsciousness, substance abuse. There was no history of any significant physical illness, trauma or head injury, visual disturbances and hearing voices.

Her premorbid functioning revealed no abnormality. In the past, there was no history suggestive of any psychiatric illness, seizures and head injury. In her family history too, nothing significant could be detected. Her menstrual cycles were regular.

On general examination, her pulse rate and blood pressure were within normal limits; nothing abnormal was detected in other parameters too. On central nervous system examination, there were no signs of raised intracranial tension or meningitis. There was no neurodeficit and no abnormality was detected in fundoscopy. No abnormality was detected in the respiratory, cardiac and gastro-intestinal system.

On mental status examination, she was looking appropriate to her stated age with her average built. She was co-operative and eye to eye contact was maintained. Rapport was established but she was preoccupied with headache. Her motor activity was decreased and reaction time increased. Her affect remained depressed. She expressed hopelessness and worry for her family members. No delusion could be elicited. There were no perceptual disturbances.

A provisional diagnosis of moderate depression with somatic symptoms was made. She was put on Escitalopram $10 \mathrm{mg}$ and Clonazepam $0.5 \mathrm{mg}$. She had mild improvement when she came for follow up after three weeks. Her dose of escitalopram was hiked to $20 \mathrm{mg}$ and clonazepam was gradually tapered and stopped. Follow-up after two months didn't show much 
improvement. This time she was investigated properly. Her hemoglobin was found to be $11.5 \mathrm{~g} \%$, total leukocyte count was $6400 / \mathrm{cu} \mathrm{mm}$, platelet count was $208 \times 10^{3} / \mathrm{mm}^{3}$. Fasting and postprandial blood sugar were $87 \mathrm{mg} / \mathrm{dl}$ and $122 \mathrm{mg} / \mathrm{dl}$ respectively. Blood urea level was $32 \mathrm{mg} \%$ and serum creatinine was $0.6 \mathrm{mg} / \mathrm{dl}$. Her sodium level was $139 \mathrm{mEq} / \mathrm{L}$ and potassium level was $4.5 \mathrm{mEq} / \mathrm{L}$. Her thyroid profile and electroencephalogram (EEG) were within normal limits. MRI of the brain with contrast revealed corticalsubcortical areas of encephalomalacia with gliosis in bilateral frontal lobes, left parietal lobe and bilateral cerebellar hemispheres which appeared to be sequelae of old insult. She was sent for consultation from a neurologist. But she didn't turn up after that.

\section{Discussion}

In our case, the patient presented with behavioural disturbances characterized by features of depression. As she didn't show much improvement with the adequate dose of the antidepressant, she was investigated further. But the MRI finding was unexpected in this particular case without any history of trauma and seizure. It has already been estimated that a significant number of patients with traumatic brain injury presented with depression and psychoses of various types. ${ }^{8}$ One study showed that after a head injury, psychoses usually had a gradual onset and a subacute or chronic course. Paranoid delusions and auditory hallucinations were the predominant features. The cases presented with schizophrenia-like psychosis had more widespread brain damage on neuroimaging, especially in the left temporal and right parietal regions, and were more impaired cognitively. ${ }^{9}$ In our case, MRI revealed encephalomalacia with gliosis in cortical-subcortical areas of bilateral frontal lobes, left parietal lobe and bilateral cerebellar hemispheres. Study on head injury concluded that subjects with lesions limited to ventromedial frontal lobes showed more aggressive and violent behaviours compared to patients with nonfrontal head injuries. ${ }^{10}$ One interesting finding we would like to mention here that in our case no feature of psychosis in the form of delusion, hallucination and violent behaviours could be detected. We emphasize the importance of imaging of the brain and its association with psychiatric manifestations even in the absence of a history of trauma though further studies are needed with more cases to establish a correlation between encephalomalacia of various parts of the brain and type of psychiatric morbidities.

\section{References}

1. Karaman E, Isildak H, Yilmaz M, Enver O, Albayram S. Encephalomalacia in the frontal lobe: complication of the endoscopic sinus surgery. J Craniofac Surg. 2011;22(6):2374-5.

2. Le TH, Gean AD. Neuroimaging of traumatic brain injury. Mt Sinai J Med 2009;76:145-62.
3. Desai SD, Patel D, Bharani S, Kharod N. Opercular syndrome: A case report and review. J Pediatr Neurosci 2013;8:123-5.

4. Ashraf M, Irshad M, Chowdhary J, Malla RA, Akhter Y. Computed tomographic study in young epileptics in Kashmir, India. Al Ameen J Med Sci 2013;6(3):272-7.

5. Alemdar M, Iseri P, Yalug I, Kutlu H, Efendi H, Komsuoglu SS. Route learning impairment associated with encephalomalasia secondary to traumatic brain injury: a case report. Appl Neuropsychol, 2008;15:150-5.

6. Li C, Chou M, Liu C, Lai C. Antiphospholipid syndrome presenting as progressive neuropsychiatric disorders: two case reports. Neuropsychiatr Dis Treat 2013;9:739-42.

7. Waldfogel S, Field HL, Wu L. Oedipism in a patient with frontal lobe encephalomalacia. Brain Inj 1994;8:377-81.

8. Achté K, Jarho L, Kyykkä T, Vesterinen E. Paranoid disorders following war brain damage. Preliminary report. Psychopathology. 1991;24:309-15.

9. Sachdev P, Smith JS, Cathcart S. Schizophrenia-like psychosis following traumatic brain injury: a chart based descriptive and case-control study. Psychol Med. 2001;31:231-9.

10. Grafman J, Schwab K, Warden D, Pridgen A, Brown HR, Salazar AM. Frontal lobe injuries, violence, and aggression: a report of the Vietnam head injury study. Neurology. 1996;46:1231-8. 